\title{
Information-theoretic model selection for optimal prediction of stochastic dynamical systems from data
}

\author{
David Darmon* \\ Department of Military and Emergency Medicine, Uniformed Services University of the Health Sciences, Bethesda, Maryland 20814, USA \\ and The Henry M. Jackson Foundation for the Advancement of Military Medicine, Bethesda, Maryland 20817, USA
}

(Received 21 November 2017; published 16 March 2018)

\begin{abstract}
In the absence of mechanistic or phenomenological models of real-world systems, data-driven models become necessary. The discovery of various embedding theorems in the 1980s and 1990s motivated a powerful set of tools for analyzing deterministic dynamical systems via delay-coordinate embeddings of observations of their component states. However, in many branches of science, the condition of operational determinism is not satisfied, and stochastic models must be brought to bear. For such stochastic models, the tool set developed for delay-coordinate embedding is no longer appropriate, and a new toolkit must be developed. We present an information-theoretic criterion, the negative log-predictive likelihood, for selecting the embedding dimension for a predictively optimal data-driven model of a stochastic dynamical system. We develop a nonparametric estimator for the negative log-predictive likelihood and compare its performance to a recently proposed criterion based on active information storage. Finally, we show how the output of the model selection procedure can be used to compare candidate predictors for a stochastic system to an information-theoretic lower bound.
\end{abstract}

DOI: 10.1103/PhysRevE.97.032206

\section{INTRODUCTION}

When studying the dynamics of a newly encountered complex system, the best first model for the system often comes directly from measurements of the system itself. When using an observed time series from the system, a first attempt may be made to reconstruct the latent state space via a delay-coordinate embedding of the time series. From its earliest discovery [1,2], this insight has grown into the dynamical systems approach to nonlinear time series analysis [3,4], which provides a set of tools for estimating the properties of a dynamical system via partial observations of the system's overall state. This approach has found broad application in the physical [3], biological [5-7], and social [8-11] sciences, as well as recent inroads into engineered systems [12]. Delay-coordinate embedding approaches find their justification in various embedding theorems $[2,13]$. These theorems, roughly, indicate that the topological properties of a dynamical system can be recovered via a delaycoordinate embedding of a trajectory from a single state of the system. Work showing that delay-coordinate embeddings can preserve geometric properties of the dynamical system is much more recent [14]. Predictive schemes based on the delaycoordinate embedding [15-18] have been found to perform quite well for deterministic dynamical systems [19].

Despite this success, many of the typical heuristics for choosing the tuning parameters of a delay-coordinate

\footnotetext{
*david.darmon.ctr@usuhs.edu
}

Published by the American Physical Society under the terms of the Creative Commons Attribution 4.0 International license. Further distribution of this work must maintain attribution to the author(s) and the published article's title, journal citation, and DOI. embedding lead to predictively suboptimal values [20]. As a simple example, the heuristic of setting the time delay for a delay-coordinate embedding to the first minima of the estimated automutual information will lead to the worst possible predictor based on a single lag: Minimizing the mutual information between the lag and future value maximizes the predictive error. In addition, the typical heuristic approaches are not necessarily applicable to high-dimensional dynamical systems [21], which in practice are more readily modeled as stochastic dynamical systems [22-24]. Viewed as a realization from a stochastic dynamical system, approaches in both the statistical [25] and nonlinear dynamics [26] literature recommend choosing the embedding dimension (equivalently, autoregressive model order) using the mean-squared error as a criterion. However, mean-squared error, a second-order property of the dynamical system, may be inappropriate when higher-order moments contain additional information relevant for prediction.

In the statistical literature, many information-theoretic model selection criteria have been proposed, including the Akaike information criterion and the Schwarz (Bayesian) information criterion [27]. These criteria, however, require the use of a parametric model. An attractive nonparametric information-theoretic criterion was proposed in Ref. [20] for selecting the embedding dimension for a delay-coordinate embedding-based predictor of a deterministic dynamical system. They propose maximizing the estimated mutual information between the embedding vector used for prediction and the future at some time horizon, a quantity called the active information storage [28], to determine the appropriate embedding dimension. Maximizing the true active information storage with respect to the embedding parameters results in the optimal parameters for prediction at that time horizon. In addition, their proposed estimator for the active informa- 
tion storage has a single tuning parameter that can be fixed following an asymptotic argument, which is beneficial both in terms of computational speed and ease of use. They show that in practice their method chooses the embedding parameters that give optimal prediction for several synthetic and real-world systems when prediction is restricted to a nearest-neighbor regression using the delay-coordinate embedding.

Given the success of active information storage with deterministic dynamical systems and its general informationtheoretic formulation, it seems natural that this success should extend to stochastic dynamical systems. In this paper, we show that this is not the case and investigate why the procedure proposed in Ref. [20] can fail to select the optimal model order for stochastic dynamical systems. Motivated by the same information-theoretic decomposition, we propose an alternative information-theoretic criterion, the negative log-predictive likelihood, for model selection for both deterministic and stochastic dynamical systems. In Sec. II, we define the active information storage for deterministic and stochastic dynamical systems and develop some of its properties and its relationship to other information-theoretic quantities. We also present the negative log-predictive likelihood and a method for estimating it from data. In Sec. III, we demonstrate the performance of the active information storage and negative log-predictive likelihood criteria for model selection with various stochastic dynamical systems. In Sec. IV, we indicate how the negative log-predictive likelihood and an estimate of the entropy rate of a stochastic dynamical system can be used to investigate the quality of candidate predictors with respect to an informationtheoretic lower bound. Finally, we conclude and consider possible directions for improvement and use of negative logpredictive likelihood for more sophisticated forms of nonlinear time series modeling in Sec. V.

\section{INFORMATION-THEORETIC MODEL SELECTION FOR STOCHASTIC DYNAMICAL SYSTEMS}

\section{A. Stochastic dynamical systems}

In this paper, we will focus on stochastic dynamical systems with continuous observables evolving in discrete time. Since in practice data from a continuous-time system is discretely sampled, this does not lead to a great loss in the applicability of our results. However, continuous-time systems do introduce additional complications in the choice of sampling rate, prediction horizon, etc. We will discuss these issues in Sec. V. Let $\left\{X_{t}\right\}_{t \in \mathbb{Z}}$ be the real-valued observable from some stochastic dynamical system. While we consider the scalar case, the results and approaches generalize in obvious ways to vector-valued systems. We will denote the block of values from time $a$ to time $b>a,\left(X_{a}, X_{a+1}, \ldots, X_{b-1}, X_{b}\right)$, by $X_{a}^{b}$. Similarly, we denote the semi-infinite past relative to time $t,\left(\ldots, X_{t-2}, X_{t-1}\right)$, by $X_{-\infty}^{t-1}$. We will use the convention of denoting random variables by upper case Roman letters and their particular realizations by lower case Roman letters. Let the observable $\left\{X_{t}\right\}_{t \in \mathbb{Z}}$ follow the model

$$
X_{t}=T\left(X_{-\infty}^{t-1}\right)+S\left(X_{-\infty}^{t-1} ; \epsilon_{t}\right)
$$

where $\left\{\epsilon_{t}\right\}_{t \in \mathbb{Z}}$ is an independent and identically distributed white noise process and $\epsilon_{t}$ is independent of $X_{s}$ for all $s<t$. In the language of Ref. [29], the deterministic skeleton $T$ is clothed by the dynamical noise term $S$, and in the case where $S \equiv 0$, we recover a deterministic map $x_{t}=T\left(x_{-\infty}^{t-1}\right)$. Implicit in (1) is the assumption that the stochastic dynamical system is conditionally stationary [30]: The functions $T$ and $S$ do not change in time. Instead of specifying $\left\{X_{t}\right\}_{t \in \mathbb{Z}}$ by its update equation, we can alternatively specify the transition or predictive density from time $t-1$ to time $t$ by $f_{X_{t} \mid X_{-\infty}^{t-1}}\left(x_{0} \mid x_{-\infty}^{-1}\right)=$ $f\left(x_{0} \mid x_{-\infty}^{-1}\right)$, where we drop the subscripts due to the assumption of conditional stationarity. Generically, these two representations of a stochastic dynamical system are equivalent [31].

In practice, for prediction, one assumes that the dynamical system has a finite, or at least approximately finite, memory, and thus the update equation can be approximated by

$$
X_{t} \approx T\left(X_{t-p_{T}}^{t-1}\right)+S\left(X_{t-p_{S}}^{t-1} ; \epsilon_{t}\right) .
$$

This relationship holds exactly for a Markov model of order $p=\max \left\{p_{T}, p_{S}\right\}$. Since the value of $p$ is not typically known a priori, we require a model selection procedure to determine it. We turn to two possibilities next.

\section{B. Active information storage of stochastic dynamical systems}

In Ref. [20], the active information storage is presented as a model selection criterion to choose both the embedding dimension $\ell$ and time delay $\tau$ for a time-delay embeddingbased predictor of a deterministic dynamical system. We consider the case where $\tau$ is taken to be the sampling rate of the observed time series since our results do not hinge on the optimization over $\tau$. We discuss the inclusion of the time delay in Sec. V. For an observable $\left\{X_{t}\right\}_{t \in \mathbb{Z}}$, the active information storage of order $\ell$ [28] is defined as the mutual information between the next-step future $X_{t}$ of the dynamical system and the previous $\ell$ time lags of the system,

$$
\mathcal{A}(\ell)=I\left[X_{t-\ell}^{t-1} \wedge X_{t}\right] .
$$

This measure is called the active information storage in contrast to the excess entropy [32], denoted by $\mathbf{E}$, which quantifies the information shared between the semi-infinite past and semi-infinite future of the process rather than just the next-step future. Thus, it measures the information in the past active in predicting the next-step value.

By expanding (3), we see that the active information storage is equivalent to the difference between the marginal entropy $h\left[X_{t}\right]$ of the dynamical system and its $\ell$ th-order entropy rate $\bar{h}(\ell)=h\left[X_{t} \mid X_{t-\ell}^{t-1}\right]$,

$$
\begin{aligned}
\mathcal{A}(\ell) & =I\left[X_{t-\ell}^{t-1} \wedge X_{t}\right], \\
& =h\left[X_{t}\right]-\bar{h}(\ell) .
\end{aligned}
$$

The I-diagram [33] with the information atoms for $X_{t}$ and $X_{t-\ell}^{t-1}$ is shown in Fig. 1. Because $h\left[X_{t}\right]$ is unchanged by the choice of $\ell$, this immediately makes obvious the following property of active information storage: The order- $\ell$ active information storage of any dynamical system must be monotonically increasing in $\ell$, since $\bar{h}(\ell)$ is a monotonically decreasing function of $\ell$. Thus, the active information storage either (a) reaches a maximum at some $p$ and remains constant, (b) asymptotically approaches a finite $\mathcal{A}(\infty)$, (c) diverges to infinity at some finite value, or (d) asymptotically approaches an infinite $\mathcal{A}(\infty)$. The first three scenarios are shown schematically in Fig. 2. Example 


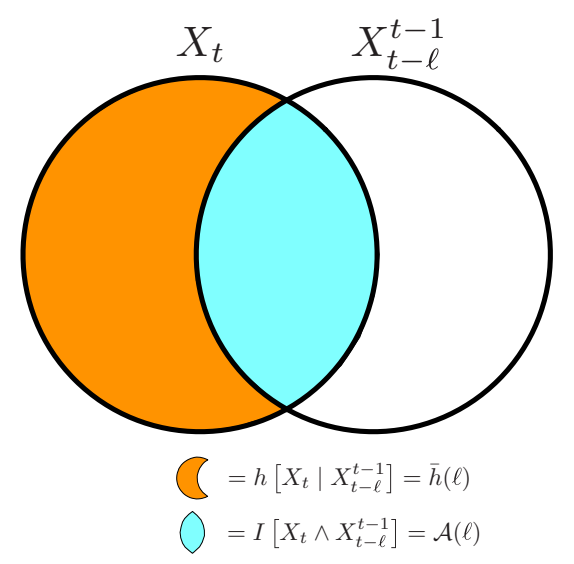

FIG. 1. The information atoms quantifying the relationship between $X_{t}$ and $X_{t-\ell}^{t-1}$. Because the order- $\ell$ entropy rate and order- $\ell$ active information storage sum to the marginal entropy $h\left[X_{t}\right]$, maximizing the active information storage is equivalent to minimizing the entropy rate.

stochastic dynamical systems with these behaviors are given next.

(a) A Markov process of order $p$ will maximize $\mathcal{A}(\ell)$ at $\ell=p$. To see this, recall that for a Markov process,

$$
\bar{h}(\ell)=h\left[X_{t} \mid X_{t-p}^{t-1}\right] \text { for all } \ell \geqslant p,
$$

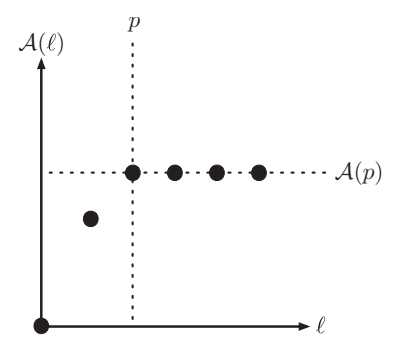

(a)

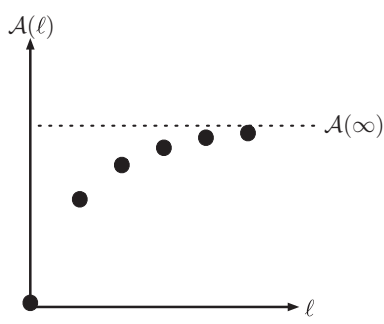

(b)

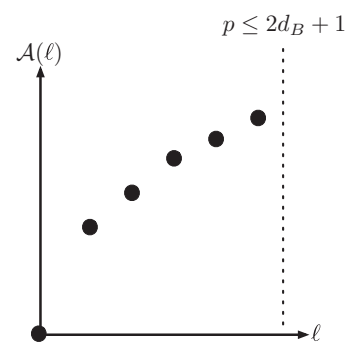

(c)

FIG. 2. Three possible behaviors of the active information storage for a dynamical system. (a) For an order- $p$ Markov model, the active information storage attains its maximum at $\ell=p$. (b) For a process with finite entropy rate but not finite Markov order, the finite past active information storage monotonically approaches the infinite past active information storage. (c) For an observation from a chaotic dynamical where the embedding theorem [13] applies, the active information storage diverges to infinity at a finite value $p \leqslant 2 d_{B}+1$, where $d_{B}$ is the box counting dimension of the attractor associated with the full state of the dynamical system. Here we use the convention that $\mathcal{A}(0)=0$. and that conditioning reduces entropy. Alternatively, apply the chain rule for mutual information and note that for $\ell>p$ we have

$$
\begin{aligned}
\mathcal{A}(\ell) & =I\left[X_{t-\ell}^{t-1} \wedge X_{t}\right], \\
& =I\left(\left[X_{t-\ell}^{t-p-1}, X_{t-p}^{t-1}\right) \wedge X_{t}\right], \\
& =I\left[X_{t-\ell}^{t-p-1} \wedge X_{t} \mid X_{t-p}^{t-1}\right]+I\left[X_{t-p}^{t-1} \wedge X_{t}\right], \\
& =I\left[X_{t-p}^{t-1} \wedge X_{t}\right]=\mathcal{A}(p),
\end{aligned}
$$

where we have $I\left[X_{t-\ell}^{t-p-1} \wedge X_{t} \mid X_{t-p}^{t-1}\right]=0$ by the $p$ th-order Markov property of the process.

(b) Any moving average process will only asymptotically approach the infinite-order active information storage. For example, the MA(1) model

$$
X_{t}=\epsilon_{t}+a \epsilon_{t-1}, t \in \mathbb{Z},
$$

with $|a|<1$ and $\left\{\epsilon_{t}\right\}_{t \in \mathbb{Z}}$ iid standard normal random variables can be rewritten as an infinite-order autoregressive model,

$$
X_{t}=\sum_{k=1}^{\infty}(-1)^{k+1} a^{k} X_{t-k}+\epsilon_{t}, t \in \mathbb{Z} .
$$

Thus, in principle, to predict $X_{t}$ optimally, we require looking infinitely far into the past. In practice, we see that the coefficients on the lagged terms decrease geometrically for $|a|<1$, and thus we expect the finite- $\ell$ active information storages to quickly approach the asymptotic value.

(c) Any chaotic system (discrete- or continuous-time) satisfying the conditions of the embedding theorem [13] will exhibit the divergence of active information storage after a sufficiently large $\ell$. For example, for the logistic map,

$$
X_{t}=r X_{t-1}\left(1-X_{t-1}\right), t \in \mathbb{Z},
$$

the "density" of $X_{t} \mid X_{t-1}=x_{t-1}$ is a point mass at $r x_{t-1}(1-$ $x_{t-1}$ ), and thus the order-1 entropy rate is equal to negative infinity. However, $X_{t}$ does have a well-defined stationary density, and thus $h\left[X_{t}\right]$ is finite. Overall, we therefore have that $\mathcal{A}(1)=\infty$ for the logistic map.

Because active information storage increases monotonically in $\ell$, in theory, it suffices to determine the first argument $\ell$ that maximizes $\mathcal{A}(\ell)$ if such an argument exists. In practice, since the stochastic dynamical system is not known, we must estimate $\mathcal{A}(\ell)$ from a particular realization or ensemble of realizations from the system. We follow [20] and use a Kraskov-Stögbauer-Grassberger (KSG) estimator for the active information storage [34]. The KSG estimator uses the relationship $I[X \wedge Y]=h[X]+h[Y]-h[X, Y]$ and a modified version of the standard $k$ th-nearest-neighbor estimator for differential entropies where the estimators for $h[X], h[Y]$, and $h[X, Y]$ are coupled through their nearest-neighbor parameter. They present two estimators for the mutual information. We follow Ref. [20] and use the second formulation of the KSG estimator. For a prescribed $\ell_{\max }$, the KSG estimator is given by

$$
\begin{aligned}
\widehat{\mathcal{A}}(\ell)= & \frac{1}{T-\ell_{\max }} \sum_{t=\ell_{\max }+1}^{T}\left\{\psi(k)-\frac{1}{k}-\psi\left(N_{\mathcal{X}^{\ell}}\left(t ; \rho_{t, k}\right)\right)\right. \\
& \left.-\psi\left(N_{\mathcal{X}}\left(t ; \rho_{t, k}\right)\right)+\psi\left(T-\ell_{\max }\right)\right\},
\end{aligned}
$$


where $\psi$ is the digamma function, $\rho_{t, k}$ is the distance to the $k$ th nearest-neighbor of $\left(X_{t-p}^{t-1}, X_{t}\right)$ under the infinity norm, and $N_{\mathcal{S}}\left(t ; \rho_{t, k}\right)$ is the number of sample points within a distance $\rho_{t, k}$ in the space $\mathcal{S}$. We use the estimator as implemented in the Java Information Dynamics Toolbox [35]. The authors of Ref. [34] state that their estimators are asymptotically unbiased for $k$ fixed and demonstrate their finite sample performances on several simulated examples. However, they do not prove that the estimators are asymptotically unbiased or, more importantly, that the estimators are consistent. See Ref. [36] for a proof that their first estimator for $I[\mathbf{X} \wedge \mathbf{Y}]$ is consistent with fixed $k$ for pairs of random vectors $(\mathbf{X}, \mathbf{Y})$ using independent and identically distributed samples from the pairs. Following the common practice, we fix $k=5$. After estimating $\hat{\mathcal{A}}(\ell)$ for $\ell=1,2, \ldots, \ell_{\max }$, we take the model order to be

$$
\ell_{\mathcal{A}}^{*}=\min \underset{\left\{1,2, \ldots, \ell_{\max }\right\}}{\arg \max } \hat{\mathcal{A}}(\ell) .
$$

In Ref. [20], the authors demonstrated that this model selection criterion chose predictively optimal model orders for predictors based on Lorenz's method of analogs [37] for both deterministic maps (logistic, Hénon) and flows (Lorenz-63, Lorenz-96). However, as we saw above, such systems attain infinite active information storage at finite values $\ell_{\mathcal{A}}^{*}$ and thus diverging $\hat{\mathcal{A}}\left(\ell_{\mathcal{A}}^{*}\right)$ in the limit of additional data. Thus, it is unclear how the model selection procedure performs for stochastic dynamical systems, where $\mathcal{A}\left(\ell_{\mathcal{A}}^{*}\right)$ may be finite. We consider the performance of (15) for stochastic dynamical systems in Sec. III.

\section{The negative log-predictive likelihood}

We next propose an alternative information-theoretic model selection criterion. As noted in the previous section, maximizing the active information storage is equivalent to minimizing the entropy rate. We therefore construct an estimator for the entropy rate following a modification of the method presented in Ref. [38] and determine the value $\ell_{\bar{h}}^{*}$ that minimizes the estimator. For a chosen $\ell_{\max }$, we estimate the order- $\ell$ entropy rate $\bar{h}(\ell)$ using the leave-window-out cross-validated negative log-predictive likelihood

$$
\hat{\bar{h}}(\theta, \ell)=-\frac{1}{T-\ell_{\max }} \sum_{t=\ell_{\max }+1}^{T} \log \hat{f}_{\theta,-t: l}\left(X_{t} \mid X_{t-\ell}^{t-1}\right),
$$

where $\hat{f}_{\theta,-t: l}$ is an estimator of the predictive density of $X_{0}$ given $X_{-\ell}^{-1}$ with tuning parameters $\theta$ leaving out the $2 l+1$ points $X_{t-p-l}^{t-l}, \ldots, X_{t-p}^{t}, \ldots, X_{t-p+l}^{t+l}$. In this paper, we take $l=0$, which corresponds to a leave-one-out cross-validated negative log-predictive likelihood. We take $\hat{f}_{\theta}$ to be the hybrid kernel density estimator- $k$-nearest-neighbor estimator, also known as a kernel nearest-neighbor estimator [39], of the predictive density. The kernel nearest-neighbor estimator of the predictive density of $X_{0}$ given $X_{-\ell}^{-1}$ is given by

$$
\hat{f}_{k, h_{f}}\left(x_{0} \mid x_{-\ell}^{-1}\right)=\frac{1}{k} \sum_{i \in N_{k}\left(x_{-\ell}^{-1}\right)} K_{h_{f}}\left(X_{i}-x_{0}\right),
$$

where $k \in\left\{1,2, \ldots, T-\ell_{\max }\right\}$ is the nearest-neighbor number, $N_{k}\left(x_{-\ell}^{-1}\right)$ is the index set such that $i \in N_{k}\left(x_{-\ell}^{-1}\right)$ if
$X_{i-\ell}^{i-1} \in\left\{X_{t-\ell}^{t-1}\right\}_{t=\ell+1}^{T}$ is one of the $k$-nearest-neighbors of $x_{-\ell}^{-1}$, $h_{f} \in(0, \infty)$ is the bandwidth for the future, and $K_{h_{f}}(x)=$ $\frac{1}{h_{f}} K\left(x / h_{f}\right)$, where $K$ is a kernel. In what follows, we take $K$ to be the Gaussian kernel. We see that (17) is precisely a regression of the synthetic data $\left\{K_{h_{f}}\left(X_{t}-x_{0}\right)\right\}_{t=\ell_{\max }+1}^{T}$ on the pasts $\left\{X_{t-\ell}^{t-1}\right\}_{t=\ell_{\max }+1}^{T}$ using a $k$-nearest-neighbor estimator to perform the regression [40]. We wish to find the $\ell$ and $\theta=\left(k, h_{f}\right)$ that minimizes (16) using the realized time series. We see that (16) will be minimized when futures assigned high density occur frequently, and those assigned low density occur infrequently. The nearest-neighbor parameter $k$ and future bandwidth $h_{f}$ will control the smoothness of the predictive density, and the model order $\ell$ will control the misspecification of the predictive density due to not accounting for enough past-future dependence. In practice, for a fixed value of $\ell$, we optimize (16) simultaneously over both $k$ and $h_{f}$ using the constrained Nelder-Mead method $[41,42]$ as implemented in the NLopt library [43], with $h_{f}$ constrained to lie in $(0, \infty)$ and $k$ constrained to lie in $\left\{1, \ldots, k_{\max }\right\}$. We consider a $k_{\max } \ll T-\ell_{\max }$ for several reasons. First, it is known that $k$-nearest-neighbor regressors are generally consistent when $k(T)$ grows faster than $\log T$ but slower than $T$ [44]. For example, taking $k(T)=\left\lceil T^{s}\right\rceil$ with any $s \in(0,1)$ would suffice. Second, for computational reasons, it is desirable to avoid an exhaustive search over all possible $k$. Thus, we take $k_{\max }=$ $\lceil\sqrt{T}\rceil$ and increase $k_{\max }$ when $k^{*}=k_{\max }$. After estimating $\hat{\bar{h}}(\ell)=\hat{\bar{h}}\left(k^{*}, h_{f}^{*}, \ell\right)$ for $\ell=1,2, \ldots, \ell_{\max }$, we take the model order to be

$$
\ell_{\bar{h}}^{*}=\min \underset{\left\{1,2, \ldots, \ell_{\max }\right\}}{\arg \min } \hat{\bar{h}}(\ell) .
$$

A Python implementation of model selection using the negative log-predictive likelihood is available through the sidpy package on GitHub [45]. We note that this criterion is closely related to a heuristic proposed in Ref. [1] for determining the dimension of a deterministic dynamical system, though that heuristic was never formally operationalized, and to the approach taken in Ref. [46]. Another related approach was proposed in Ref. [47], using a coarse-graining of the observed time series with a heuristic objective function. We compare the performance of the negative log-predictive likelihood model selection criterion to the active information storage criterion in the following section.

\section{DEMONSTRATION OF MODEL SELECTION WITH STOCHASTIC MAPS}

Because of the relationship (5) between active information storage and entropy rate, it would initially seem reasonable that the order criteria $\ell_{\mathcal{A}}^{*}$ and $\ell_{\bar{h}}^{*}$ would give the same answer for stochastic dynamical systems, especially in the limit of long time series. In the following sections, we demonstrate that this is not the case, and that they can in fact give diverging answers in the limit of long time series, with $\ell_{\mathcal{A}}^{*}$ inconsistent and $\ell_{\bar{h}}^{*}$ consistent for the true model order.

\section{A. Stochastic logistic map}

As the first example, we consider the logistic map clothed in dynamical noise. Consider the stochastic process generated 
by the stochastic difference equation

$$
X_{t}=3.68 X_{t-1}\left(1-X_{t-1}\right)+0.4 \epsilon_{t},
$$

where the dynamical noise $\left\{\epsilon_{t}\right\}$ is taken to be independent and identically distributed with the same distribution as the sum of 48 independent and identically distributed uniform random variables with support on $[-1 / 64,1 / 64]$. Thus $\epsilon_{t}$ has mean 0 and variance $1 / 256$ with bounded support [-3/4,3/4] and by the Berry-Essèen theorem [48] is approximately normally distributed. The bounded support for $\epsilon_{t}$ is necessary to ensure that the stochastic process generated by (19) is stationary. See Ref. [49] for additional details on this technical requirement. Note that (19) is equivalent to the deterministic logistic map $x_{t}=r x_{t-1}\left(1-x_{t-1}\right)$ clothed with dynamical noise and that the deterministic logistic map with $r=3.68$ is chaotic. Noting that (19) is a first-order Markov model, we know that $\mathcal{A}(\ell)=\mathcal{A}(1)$ and $\bar{h}(\ell)=\bar{h}(1)$ for all values of $\ell$. Moreover, the entropy rate can be computed from the entropy of the dynamical noise $0.4 \epsilon_{t}$. In particular, the specific entropy rate [38] of the dynamical system is given by $h\left[X_{t} \mid X_{t-1}=x\right]=h\left[0.4 \epsilon_{t}\right]$ for all values of $x$. Thus, averaging over $X_{t-1}$, we have that the entropy rate is also given by $h\left[0.4 \epsilon_{t}\right]$. The density of $\epsilon_{t}$ is given by the 48 -fold convolution of $32 \cdot \mathbb{1}_{[-1 / 64,1 / 64]}(x)$, whose entropy we compute using PaCAL [50]. This gives $\bar{h}(1)=h\left[0.4 \epsilon_{t}\right]=$ $-2.2699543 \ldots$ To determine the active information storage, we recall that $\mathcal{A}(1)=h\left[X_{t}\right]-\bar{h}(1)$. Thus, we require the entropy $h\left[X_{t}\right]$ of the stationary density of the dynamical system. We approximate the stationary density using the ChapmanKolmogorov relation method given in Ref. [29] and its entropy via Gaussian quadrature, giving $h\left[X_{t}\right]=-0.291922 \ldots$, and thus $\mathcal{A}(1)=1.97803 \ldots$

We generate $S=100$ realizations of length $T=$ $10^{2}, 10^{3}, 10^{4}$, and $10^{5}$ from (19) and compute the estimates $\widehat{\mathcal{A}}(\ell)$ and $\hat{\bar{h}}(\ell)$ for $\ell=1, \ldots, 5$ using the estimators described in Secs. II B and II C. We begin by considering their marginal sampling distributions as a function of $\ell$. Figures 3(a) and 3(b) show their distributions (sample mean \pm sample standard deviation) using the $S=100$ simulations for the differing lengths of time series, along with the exact values of $\mathcal{A}(\ell)$ and $\bar{h}(\ell)$. For small to moderate length time series, the active information storage estimator (negative log-predictive likelihood estimator) provide negatively (positively) biased estimates with the bias increasing in $\ell$. This is not surprising, given the challenge of the curse of dimensionality for estimation problems in general and work showing how the bias of the KSG estimator scales with the dimension of the estimation problem in particular [36]. For long time series $\left(T=10^{5}\right)$, the bias becomes positive, rather than negative, for $\hat{\mathcal{A}}(1), \hat{\mathcal{A}}(2)$, and $\hat{\mathcal{A}}(3)$. In contrast, with this length of time series, the bias remains negative for all estimators $\hat{\bar{h}}(\ell)$, with the bias continuing to decrease with increasing $T$.

Of course, for model selection, the marginal sampling distributions of $\widehat{\mathcal{A}}(\ell)$ and $\hat{\bar{h}}(\ell)$ are not of interest; instead, how they vary jointly determines the model orders selected by $\ell_{\mathcal{A}}^{*}$ and $\ell_{\bar{h}}^{*}$. We next consider how $\ell_{\mathcal{A}}^{*}$ and $\ell_{\bar{h}}^{*}$ behave across the 100 realizations. The results are summarized in Table I. The active information storage criterion chooses the correct value of $\ell_{A}^{*}=1$ for $100 \%, 100 \%, 81 \%$, and $3 \%$ of the realizations of the length $T=10^{2}, 10^{3}, 10^{4}$, and $10^{5}$ time series, respectively,
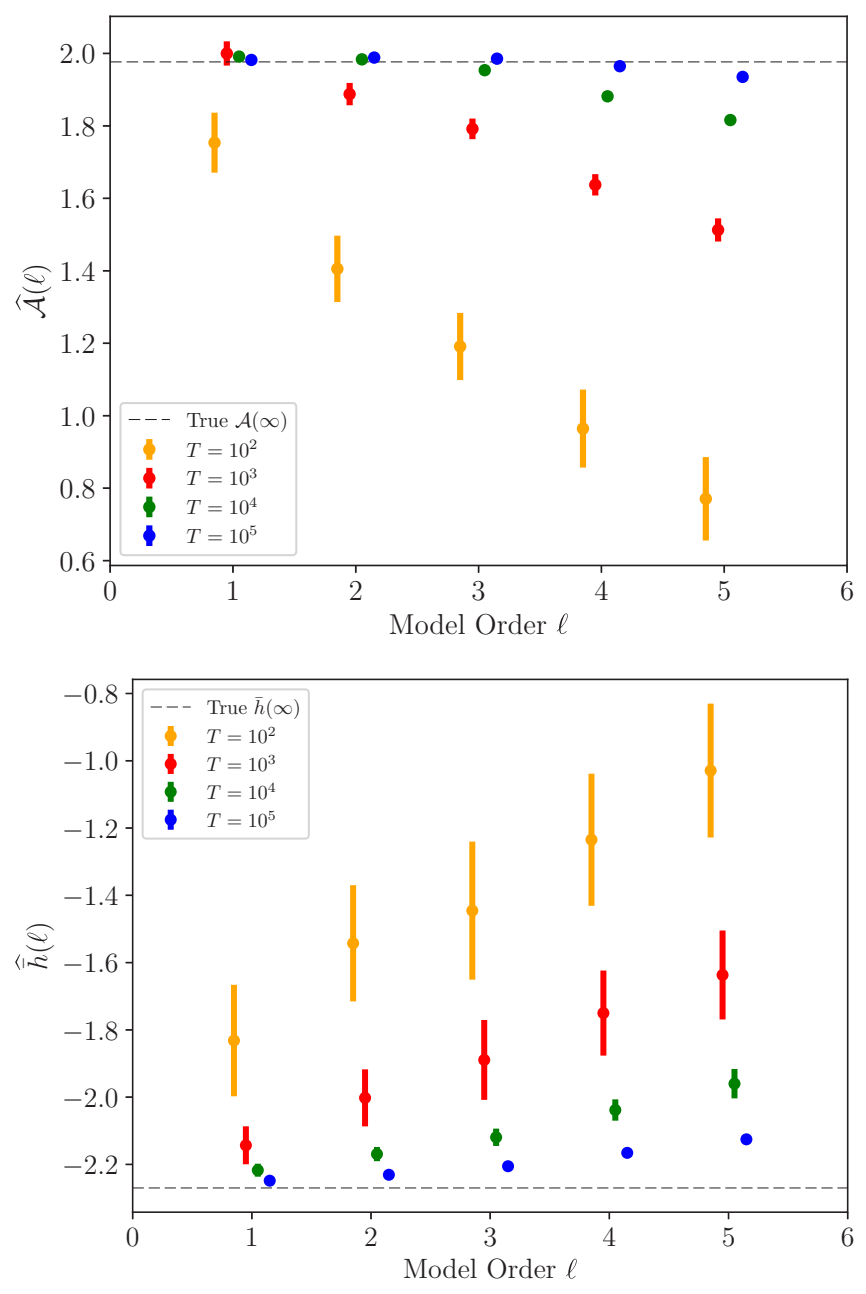

FIG. 3. The sampling distribution for the active information storage estimator with $k=5$ ( $\hat{\mathcal{A}}$, top) and the negative log-predictive likelihood estimator ( $\hat{\bar{h}}$, bottom) using $S=100$ realizations from the stochastic logistic map for varying time series lengths. The figures show the sample mean \pm the sample standard deviation of the $S=100$ realizations. The dashed horizontal lines correspond to the exact active information storage (top) and entropy rate (bottom) for the stochastic logistic map.

while the negative log-predictive likelihood chooses $\ell_{\bar{h}}^{*}=1$ for $99 \%, 100 \%, 100 \%$, and $100 \%$ of the realizations. Thus, overall, the active information storage criterion performs worse with increasing time series length, while the negative log-predictive likelihood criterion chooses the correct model order almost perfectly. It is perhaps initially counterintuitive that the active information storage criterion performs better for shorter time series than longer ones. However, again considering Fig. 3(a); we see that this is entirely a consequence of the negative bias being much larger for larger $\ell$ with shorter time series, since the true value of the active information storage is constant for all values of $\ell$. In Table I and all additional tables with estimated proportions, a rough $95 \%$ confidence intervals for the proportions is given by considering the estimated proportions \pm 0.1 . This width is from the normal approximation for the estimated proportion of a binomial distribution in the extreme case that the estimated proportion is $1 / 2$ using $S=100$ draws. 
TABLE I. The proportion of the 100 realizations of the stochastic logistic map given by (19) for which $\ell^{*}$ was chosen as a function of the time series length using (a) the active information storage selection criterion and (b) the negative log-predictive likelihood criterion.

(a)

\begin{tabular}{lccccc} 
& \multicolumn{5}{c}{ Proportion with $\ell_{\mathcal{A}}^{*}=$} \\
\cline { 2 - 6 }$T$ & 1 & 2 & 3 & 4 & 5 \\
\hline $10^{2}$ & $\mathbf{1 . 0 0}$ & 0.00 & 0.00 & 0.00 & 0.00 \\
$10^{3}$ & $\mathbf{1 . 0 0}$ & 0.00 & 0.00 & 0.00 & 0.00 \\
$10^{4}$ & $\mathbf{0 . 8 1}$ & 0.19 & 0.00 & 0.00 & 0.00 \\
$10^{5}$ & $\mathbf{0 . 0 3}$ & 0.86 & 0.11 & 0.00 & 0.00
\end{tabular}

(b)

Proportion with $\ell_{\bar{h}}^{*}=$

\begin{tabular}{lccccc}
\cline { 2 - 6 }$T$ & 1 & 2 & 3 & 4 & 5 \\
\hline $10^{2}$ & $\mathbf{0 . 9 9}$ & 0.01 & 0.00 & 0.00 & 0.00 \\
$10^{3}$ & $\mathbf{1 . 0 0}$ & 0.00 & 0.00 & 0.00 & 0.00 \\
$10^{4}$ & $\mathbf{1 . 0 0}$ & 0.00 & 0.00 & 0.00 & 0.00 \\
$10^{5}$ & $\mathbf{1 . 0 0}$ & 0.00 & 0.00 & 0.00 & 0.00 \\
\hline \hline
\end{tabular}

For proportions away from $1 / 2$, narrower confidence intervals would result.

\section{B. Self-exciting threshold autoregressive model}

As a second example, we consider a stochastic dynamical system from the class of self-exciting threshold autoregressive (SETAR) models proposed in Ref. [51]. SETAR models are simple piecewise linear autoregressive models that capture many characteristics of nonlinear time series including subharmonics, amplitude-frequency dependence, and time irreversibility. The general form of a $\operatorname{SETAR}\left(k ; p_{1}, \ldots, p_{k}\right)$ model with $k$ regimes where each regime $i$ has model order $p_{i}$ is

$X_{t}=\sum_{i=1}^{k}\left\{b_{i 0}+b_{i 1} X_{t-1}+\ldots+b_{i p_{i}} X_{t-p_{i}}+\sigma_{i} \epsilon_{t}\right\} I_{A_{i}}\left(X_{t-d}\right)$,

where $I_{A}$ is the indicator function for the set $A$ and the sets $\left\{A_{i}\right\}_{i=1}^{k}$ are a partition of the real line. For example, the stochastic tent map is a $\operatorname{SETAR}(2 ; 1,1)$ model. We consider the particular $\operatorname{SETAR}(2 ; 2,2)$ model of the Canadian lynx data [52] presented in Ref. [29] as a minimal model that captures many of the observed features of the log-population over time, including the asymmetry in length of the population growth-decay periods and the typical period for a population cycle. The $\operatorname{SETAR}(2 ; 2,2)$ model is given by

$$
X_{t}=\left\{\begin{array}{ll}
0.62+1.25 X_{t-1}-0.43 X_{t-2}+\epsilon_{-, t} & : X_{t-2} \leqslant 3.25 \\
2.25+1.52 X_{t-1}-1.24 X_{t-2}+\epsilon_{+, t} & : X_{t-2}>3.25
\end{array},\right.
$$

where $\left\{\epsilon_{-, t}\right\}$ and $\left\{\epsilon_{+, t}\right\}$ are independent and identically distributed normal random variables with variances 0.0381 and 0.0626 , respectively. The entropy rate and active information for this process are $\bar{h}(\infty)=\bar{h}(2) \approx-0.14$ and $\mathcal{A}(\infty)=$ $\mathcal{A}(2) \approx 1.01$. See Appendix for the procedure for approximating these values.
TABLE II. The proportion of the 100 realizations from the $\operatorname{SETAR}(2 ; 2,2)$ model given by $(21)$ for which $\ell^{*}$ was chosen to be $1, \ldots, 5$ as a function of the time series length using (a) the active information storage selection criterion and (b) the negative log-predictive likelihood criterion.

(a)

\begin{tabular}{lccccc} 
& \multicolumn{5}{c}{ Proportion with $\ell_{\mathcal{A}}^{*}=$} \\
\cline { 2 - 6 }$T$ & 1 & 2 & 3 & 4 & 5 \\
\hline $10^{2}$ & 0.02 & $\mathbf{0 . 7 1}$ & 0.22 & 0.04 & 0.01 \\
$10^{3}$ & 0.00 & $\mathbf{0 . 9 7}$ & 0.03 & 0.00 & 0.00 \\
$10^{4}$ & 0.00 & $\mathbf{0 . 9 6}$ & 0.04 & 0.00 & 0.00 \\
$10^{5}$ & 0.00 & $\mathbf{0 . 7 2}$ & 0.28 & 0.00 & 0.00
\end{tabular}

(b)

\begin{tabular}{lccccc} 
& \multicolumn{5}{c}{ Proportion with $\ell_{\bar{h}}^{*}=$} \\
\cline { 2 - 6 }$T$ & 1 & 2 & 3 & 4 & 5 \\
\hline $10^{2}$ & 0.00 & $\mathbf{0 . 8 0}$ & 0.19 & 0.00 & 0.01 \\
$10^{3}$ & 0.00 & $\mathbf{1 . 0 0}$ & 0.00 & 0.00 & 0.00 \\
$10^{4}$ & 0.00 & $\mathbf{1 . 0 0}$ & 0.00 & 0.00 & 0.00 \\
$10^{5}$ & 0.00 & $\mathbf{1 . 0 0}$ & 0.00 & 0.00 & 0.00 \\
\hline \hline
\end{tabular}

We again consider $S=100$ realizations of length $T=$ $10^{2}, 10^{3}, 10^{4}$, and $10^{5}$ from the $\operatorname{SETAR}(2 ; 2,2)$. The fraction of realizations for which $\ell^{*}$ was chosen to be $1, \ldots, 5$ of the 100 realizations is given in Table II. The active information storage initially chooses the correct value of $\ell^{*}=2$ among $71 \%, 97 \%$, and $96 \%$ of the samples. However, as with the stochastic logistic map, the model selector is inconsistent for $T=10^{5}$, choosing the correct model order only in $72 \%$ of the samples and $\ell^{*}=3$ for the remaining $28 \%$ of the samples. As with the stochastic logistic map, the noncomparable biases in the active information storage estimator for varying $\ell$ cause it to fail with increasing $T$, as demonstrated in Fig. 4(a). In contrast, the negative log-predictive likelihood estimator chooses the correct model order over $80 \%$ of the time for $T=$ $10^{2}$, and correctly specifies the model order for all samples with $T>10^{2}$. This is again because the leave-window-out cross-validated negative log-predictive likelihood determines the optimal model without overfitting, as demonstrated in Fig. 4(b).

\section{Autoregressive conditionally heteroskedastic model}

As our final example, we consider the autoregressive conditionally heteroskedastic (ARCH) model. Such models are popular for financial time series, where the conditional variance is known to increase as the time series deviates from baseline and therefore exhibit volatility. A general $\operatorname{ARCH}(p)$ model is given by

$$
X_{t}=\sqrt{b_{0}+\sum_{j=1}^{p} b_{j} X_{t-j}^{2}} \cdot \epsilon_{t},
$$

where $b_{j} \geqslant 0$ and $\left\{\epsilon_{t}\right\}$ are taken to be independent and identically distributed with mean 0 and variance 1 and independent of $X_{t-k}$ for $k \geqslant 1$. Clearly, because of the independence between $\left\{X_{t-k}\right\}_{k \geqslant 1}$ and $\epsilon_{t}$, we have that $E\left[X_{t}\right]=0$ and $E\left[X_{t} \mid X_{t-\ell}^{t-1}\right]=$ 0 for all $\ell \geqslant 1$. Thus, the $\operatorname{ARCH}(p)$ model is a white noise process: A stochastic process where the autocorrelations are 

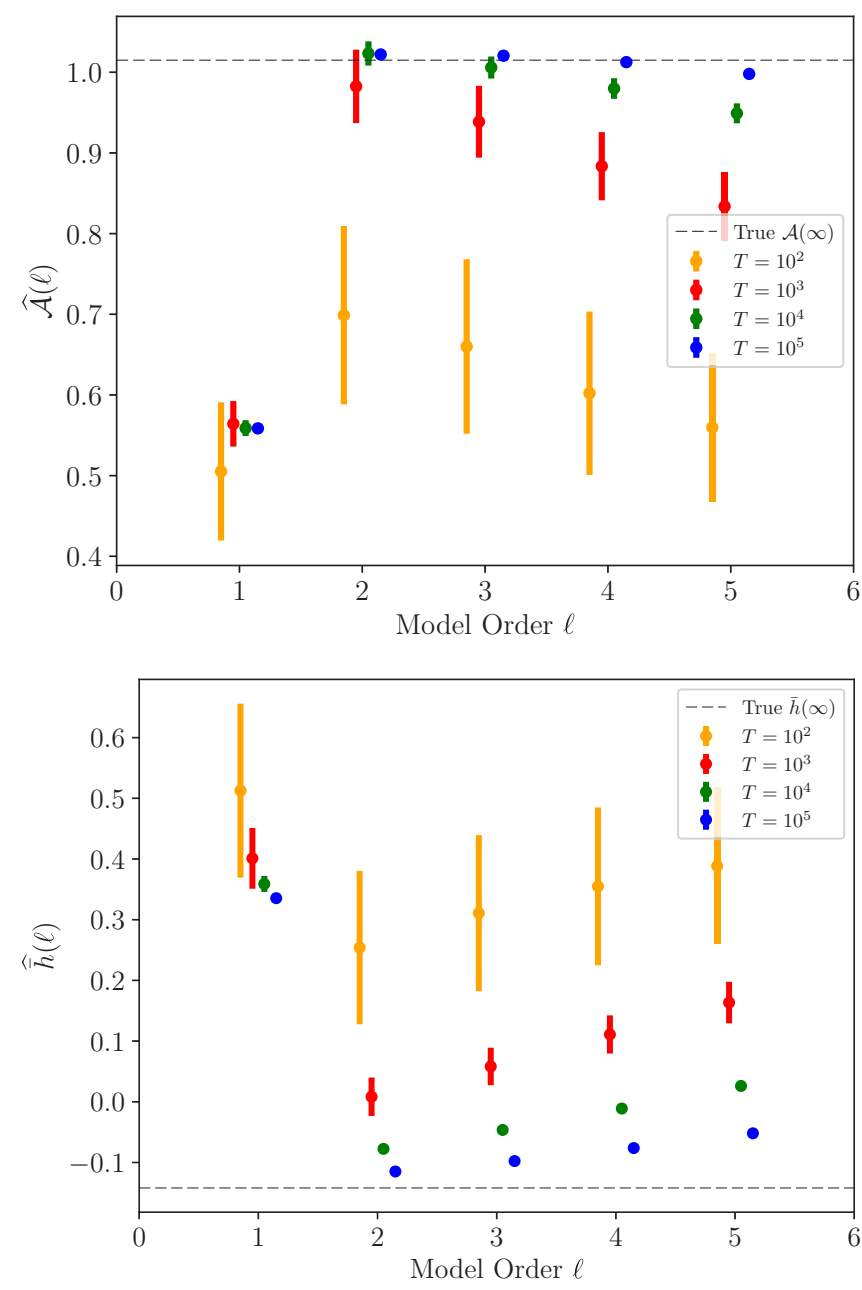

FIG. 4. The sampling distribution for the active information storage estimator with $k=5$ ( $\hat{\mathcal{A}}$, top) and the negative log-predictive likelihood estimator ( $(\hat{\bar{h}}$, bottom) using $S=100$ realizations from the $\operatorname{SETAR}(2 ; 2,2)$ model for varying time series lengths.

zero for all possible time shifts. This implies that the optimal predictor in the mean-squared sense is identically 0 , and thus any mean-squared error model selection criteria, like the Ragwitz criterion [26], would choose $\ell^{*}=0$. However, even though the autocorrelation is zero for all possible time shifts, the autoassociations, captured by higher-order moments of the process, are not identically zero. Thus, $\ell=0$ is clearly not the optimal model order for a general predictor of the process, since an $\operatorname{ARCH}(p)$ model is a Markov process of order $p$. An elaborate machinery for estimating the model order of $\operatorname{ARCH}(p)$ process exists. See, for example, Chapter 4 of Ref. [40]. However, this machinery relies on knowing the model is in the ARCH class a priori. A model-free informationtheoretic approach would be desirable. Clearly, as a Markov process, the active information storage (entropy rate) will be maximized (minimized) at the model order $\ell=p$.

We consider $S=100$ realizations of length $T=$ $10^{2}, 10^{3}, 10^{4}$, and $10^{5}$ from the $\mathrm{ARCH}(1)$ model

$$
X_{t}=\sqrt{0.5+0.5 X_{t-1}^{2}} \cdot \epsilon_{t}
$$

TABLE III. The proportion of the 100 realizations from the ARCH(1) model given by (23) for which $\ell^{*}$ was chosen to be $1, \ldots, 5$ as a function of the time series length using (a) the active information storage selection criterion and (b) the negative logpredictive likelihood criterion

(a)

\begin{tabular}{cccccc} 
& \multicolumn{5}{c}{ Proportion with $\ell_{\mathcal{A}}^{*}=$} \\
\cline { 2 - 6 }$T$ & 1 & 2 & 3 & 4 & 5 \\
\hline $10^{2}$ & $\mathbf{0 . 2 9}$ & 0.14 & 0.17 & 0.23 & 0.17 \\
$10^{3}$ & $\mathbf{0 . 4 5}$ & 0.22 & 0.08 & 0.16 & 0.09 \\
$10^{4}$ & $\mathbf{0 . 6 4}$ & 0.24 & 0.05 & 0.06 & 0.01 \\
$10^{5}$ & $\mathbf{0 . 6 9}$ & 0.22 & 0.08 & 0.01 & 0.00
\end{tabular}

(b)

\begin{tabular}{cccccc} 
& \multicolumn{5}{c}{ Proportion with $\ell_{\bar{h}}^{*}=$} \\
\cline { 2 - 6 }$T$ & 1 & 2 & 3 & 4 & 5 \\
\hline $10^{2}$ & $\mathbf{0 . 3 6}$ & 0.25 & 0.12 & 0.16 & 0.11 \\
$10^{3}$ & $\mathbf{0 . 7 5}$ & 0.20 & 0.03 & 0.01 & 0.01 \\
$10^{4}$ & $\mathbf{0 . 8 9}$ & 0.10 & 0.01 & 0.00 & 0.00 \\
$10^{5}$ & $\mathbf{0 . 9 3}$ & 0.07 & 0.00 & 0.00 & 0.00 \\
\hline \hline
\end{tabular}

where $\epsilon_{t}$ is a standard normal random variable. The fraction of realizations for which $\ell^{*}$ was chosen to be $1,2, \ldots, 5$ of the 100 realizations is given in Table III. The active information storage procedure chooses the correct model less than $70 \%$ of the time for each time series length, though unlike the previous examples, the proportion does improve with increasing time series length. In contrast, the negative log-predictive likelihood estimator chooses the correct model order over $70 \%$ of the time for $T>10^{2}$, and only misspecifies seven realizations for $T=10^{5}$. Thus again we see the benefit of treating the choice of $\ell^{*}$ directly as a model selection problem.

\section{THE UTILITY OF ENTROPY RATE IN EVALUATING CANDIDATE PREDICTORS}

The authors of Ref. [20] suggest that the active information storage may provide a useful measure of the amount of information the past provides about the next step future, regardless of the predictive method employed. This concept can be directly operationalized using the entropy rate. The continuous version of Fano's inequality provides a lower bound for the mean-squared error of any autoregressive predictor $g\left(X_{-\infty}^{t-1}\right)$ of the future $X_{t}$ [53]. In particular, the mean-squared error is bounded below by an exponential in the entropy rate $\bar{h}=\bar{h}(\infty)$ of the stochastic dynamical system,

$$
E\left[\left(X_{t}-g\left(X_{-\infty}^{t-1}\right)\right)^{2}\right] \geqslant \frac{e^{2 \bar{h}}}{2 \pi e} .
$$

Thus, with an estimate of the entropy rate as well as an estimate of the mean-squared error for a predictor $g$, one can determine how close to this error floor one has come. The optimal mean-squared error will be achieved by the conditional mean of $X_{t}$ given $X_{-\infty}^{t-1}, m\left(X_{-\infty}^{t-1}\right)=E\left[X_{t} \mid X_{-\infty}^{t-1}\right]$. For any finite $\ell$, we can consider the mean-squared error optimal predictor $m\left(X_{t-\ell}^{t-1}\right)=E\left[X_{t} \mid X_{t-\ell}^{t-1}\right]$. In principle, $m\left(X_{t-\ell}^{t-1}\right)$ can be estimated using a fully nonparametric method such as $k$-nearest-neighbor or kernel regression. However, because of 
the curse of dimensionality, it may not be possible to estimate the conditional mean well with the data set in hand, and other approximate models may be useful, balanced at a better point between bias and variance. We propose a combined model selection and comparison procedure to choose among candidate predictors.

The proposed method proceeds as follows. First, choose the appropriate model order $\ell_{\bar{h}}^{*}$ by minimizing the negative log-predictive likelihood as described in Sec. II C. Next, using this value of $\ell_{\bar{h}}^{*}$, estimate the order- $\ell_{\bar{h}}^{*}$ entropy rate $\hat{\bar{h}}=\hat{\bar{h}}\left[X_{t} \mid\right.$ $X_{t-\ell_{h}^{*}}^{t-1}$. Using the estimated entropy rate $\hat{\bar{h}}$, compute the lower bound of the mean-squared error using (24). Finally, from a collection of $C$ candidate predictive models $\left\{\hat{g}_{c}\right\}_{c=1}^{C}$, compute the mean-squared error where the data have been split into a training or estimation half and a testing or evaluation half. The discrepancy between the mean-squared error lower bound provided by $\hat{\bar{h}}$ and the empirical estimates of the mean-squared errors for each candidate predictor provide an indication of which predictor comes closest to the information-theoretic lower bound.

The estimate of the entropy rate $\hat{\bar{h}}$ can be taken as the value of the negative log-predictive likelihood used for model selection, which provides an indirect estimator of the entropy rate, or from a direct estimator of the entropy rate. We use the second approach, noting the observed positive bias of the negative log-predictive likelihood for the entropy rate in the logistic and SETAR cases in Sec. III. Recalling that $\bar{h}(\ell)=h\left[X_{t-\ell}^{t-1}, X_{t}\right]-$ $h\left[X_{t-\ell}^{t-1}\right]$, an estimate for $\bar{h}(\ell)$ may be obtained by estimating each entropy separately and taking their difference. For each entropy, we use the $k$ th-nearest-neighbor estimator [54]. Given a sample $\left\{\mathbf{X}_{i}\right\}_{i=1}^{n}$ of $d$-dimensional random vectors, the $k$ thnearest-neighbor estimator of the entropy of $\mathbf{X}$ is given by

$$
\hat{h}[\mathbf{X}]=\log (n)-\psi(k)+\log V_{d, q}+\frac{d}{n} \sum_{i=1}^{n} \log \rho_{i, k, q},
$$

where $\rho_{i, k, q}$ is the distance from $\mathbf{X}_{i}$ to its $k$ th-nearest-neighbor among $\left\{\mathbf{X}_{j}\right\}_{j \neq i}$ under the $L^{q}$-norm $\|\mathbf{x}\|_{q}=\left(\sum_{j=1}^{d}\left|x_{j}\right|^{q}\right)^{1 / q}$, and $V_{d, q}$ is the volume of the unit $d$-ball under the $L^{q}$ norm, $V_{d, q}=\frac{\left[2 \Gamma\left(\frac{1}{q}+1\right)\right]^{d}}{\Gamma\left(\frac{d}{q}+1\right)}$. In the following, we take $q=2$, the Euclidean norm. Using (25) for each of the entropies gives the entropy rate estimator $\hat{\bar{h}}=\hat{h}(\ell)=\hat{h}\left[X_{t-\ell}^{t-1}, X_{t}\right]-\hat{h}\left[X_{t-\ell}^{t-1}\right]$.

In the following sections, we demonstrate this method with two systems: the stochastic logistic map given in Sec. III A and a projection of the state vector from a stochastically forced Lorenz-63 system. In both cases, we consider three families of predictors:

Linear Autoregressive Predictor: For the linear autoregressive model, we use the predictor

$$
g_{\mathrm{LAR}}\left(x_{1}^{\ell^{*}}\right)=\mu+\beta^{T} x_{1}^{\ell^{*}},
$$

where the parameters $\mu$ and $\beta$ are estimated via ordinary least squares.

Additive Autoregressive Predictor: The linear autoregressive model is clearly very restrictive in its form. A natural relaxation is given by the additive autoregressive model [55],

$$
g_{\mathrm{AAR}}\left(x_{1}^{\ell^{*}}\right)=\mu+\sum_{j=1}^{\ell^{*}} f_{j}\left(x_{j}\right)
$$

where we allow an additive decomposition of the drift portion of the stochastic dynamical system in terms of nonlinear functions $\left\{f_{j}\right\}_{j=1}^{\ell *}$ of each lagged variable. Each additive term $f_{j}$ is estimated using splines via the backfitting algorithm where the smoothing parameters for the splines are chosen by generalized cross-validation on the training set [56].

$k$-Nearest-neighbor predictor: For a fully nonparametric predictor, we consider a $k$-nearest-neighbor regression

$$
g_{k \mathrm{NN}}\left(x_{1}^{\ell^{*}}\right)=\frac{1}{k} \sum_{i \in N_{k}\left(x_{1}^{\ell^{*}}\right)} X_{i}
$$

where $N_{k}\left(x_{1}^{\ell^{*}}\right)$ is the index set as described in Sec. IIC. This is identical to the Lorenz's method of analogs predictor used in Ref. [20] to assess the validity of active information storage as a model selection criterion where they considered $k=1$, and the predictor used for the Ragwitz criterion [26] where typically $k \approx 5$. These choices for $k$ will generally lead to suboptimal prediction even for deterministic systems. We therefore consider $k=k^{*}$, where $k^{*}$ is chosen to minimize the leave-one-out-cross-validated mean-squared error on the training data.

This list is clearly not exhaustive. For a more comprehensive list of candidate predictors, see Ref. [40]. For each system, we generate $S=100$ realizations of length $T=2 \times 10^{4}$. We use the first half of the data to choose the model order $\ell_{\bar{h}}^{*}$, estimate the entropy rate $\hat{\bar{h}}\left(\ell_{\bar{h}}^{*}\right)$, and estimate the predictors $\left\{\hat{g}_{c}\right\}$. We use the second half of the data to estimate the out-of-sample mean-squared error using $\left\{\hat{g}_{c}\right\}$.

We first consider the stochastic logistic map studied in Sec. III A, given by (19). The update equation for the stochastic logistic map falls into the class of additive autoregressive models with $f_{1}\left(x_{1}\right)=3.68 x_{1}\left(1-x_{1}\right)$. Thus, one might a priori expect the additive autoregressive predictor to perform best. The model selection procedure chooses $\ell_{\bar{h}}^{*}=1$ for all of the realizations. Figure 5 shows Tukey box plots [57] of the mean-squared errors relative to the floor determined by the estimated entropy rate, $\hat{E}_{\mathrm{MSE}, c}-\frac{e^{2 \hat{h}}}{2 \pi e}$. We see that both the additive autoregressive and $k$-nearest-neighbor predictors drastically outperform the linear autoregressive predictor, with median relative meansquared errors of $1.27 \times 10^{-4}$ and $1.07 \times 10^{-4}$, respectively, compared to $2.76 \times 10^{-2}$. The $k$-nearest-neighbor predictor outperforms the additive autoregressive predictor, despite the system falling into the latter's model class, likely due to suboptimality of cross-validation for tuning parameters in the presence of autocorrelated data [58]. Both, however, approach the information-theoretic floor for the mean-squared error.

We next consider the Lorenz-1963 system [59] forced by additive stochastic noise, given by the system of stochastic differential equations

$$
\begin{aligned}
d X_{t} & =\sigma\left(Y_{t}-X_{t}\right) d t+\eta_{X} d W_{X, t}, \\
d Y_{t} & =\left(X_{t}\left(\rho-Z_{t}\right)-Y_{t}\right) d t+\eta_{Y} d W_{Y, t}, \\
d Z_{t} & =\left(X_{t} Y_{t}-\beta Z_{t}\right) d t+\eta_{Z} d W_{Z, t},
\end{aligned}
$$




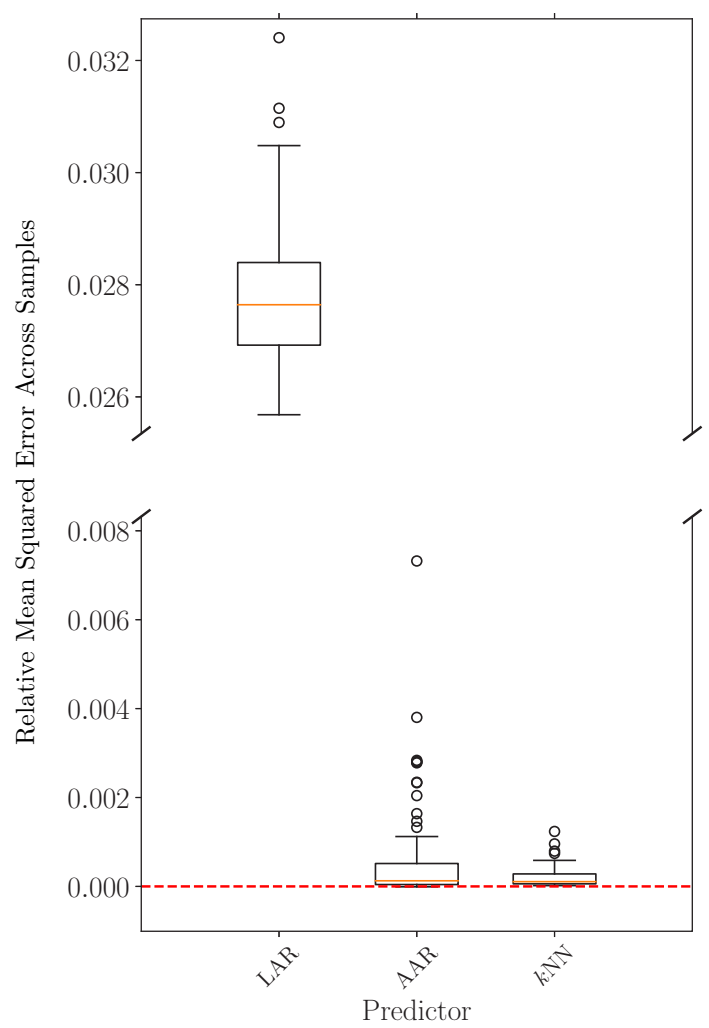

FIG. 5. The mean-squared errors for the linear autoregressive (LAR), additive autoregressive (AAR), and $k$-nearest-neighbor $(k \mathrm{NN})$ predictors relative to the floor determined by the estimated entropy rate, $\hat{E}_{\mathrm{MSE}, c}-\frac{e^{2 \hat{h}}}{2 \pi e}$, for the stochastic logistic map across 100 realizations. Note the break in the vertical axis.

with $\sigma=10, \beta=8 / 3$, and $\rho=28 ; W_{X, t}, W_{Y, t}$, and $W_{Y, t}$ uncorrelated standard Brownian motions; and dynamical noise standard deviations $\eta_{X}=\eta_{Y}=\eta_{Z}=2$. The attractor of the deterministic Lorenz system has a box counting dimension of approximately 2.06 [60], so an application of the embedding theorem from [13] implies that a model order of $\ell^{*}=5$ suffices for perfect prediction of the observation of a single state on the attractor. However, note that for a delay-based predictor from a scalar observation of a stochastic dynamical system, while the overall system is Markovian, the individual states are generally nonlinear autoregressive moving average (NLARMA) processes [61], much as the marginals of a linear vector autoregressive process are generally linear vector autoregressive moving average (ARMA) processes. Thus, the model order $\ell_{\bar{h}}^{*}$ determined by the negative logpredictive likelihood determines a finite-order approximation for the NLARMA process appropriate for the amount of data available.

We generate 100 realizations from (29) using the SRI2 stochastic integrator from Ref. [62] with step size $h=$ 0.05 , and consider the $X_{t}$ state variable with a time delay of $\tau=0.1$. That is, we consider predictors for $X_{t}$ of the form $\left(X_{t-\ell \tau}, \ldots, X_{t-2 \tau}, X_{t-\tau}\right)$ with $\tau=0.1$. The model selection procedure chooses $\ell_{\bar{h}}^{*}=4$ for all of the realizations. Figure 6 shows the relative mean-squared errors for each of the predictors. As with the stochastic logistic map, the linear

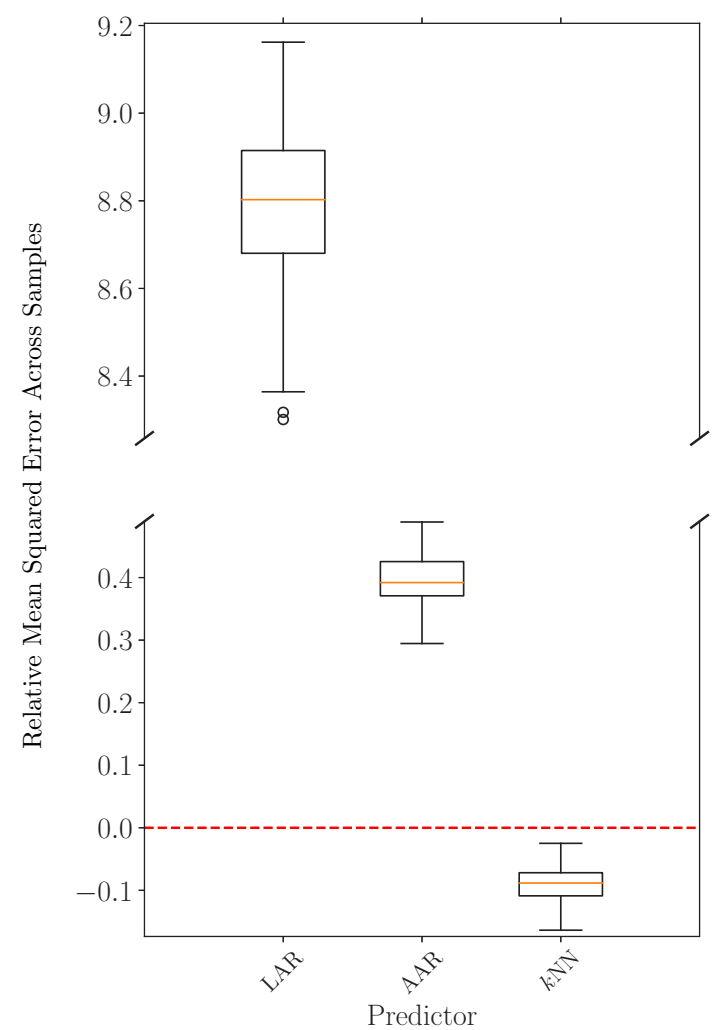

FIG. 6. The mean-squared errors for the linear autoregressive (LAR), additive autoregressive (AAR), and $k$-nearest-neighbor $(k \mathrm{NN})$ predictors relative to the floor determined by the estimated entropy rate, $\hat{E}_{\mathrm{MSE}, c}-\frac{e^{2 \hat{h}}}{2 \pi e}$, for the stochastic Lorenz system across 100 realizations. Note the break in the vertical axis.

autoregressive predictor performs worst, with a median relative mean-squared error of 8.8 , followed by the additive autoregressive predictor with 0.39 and the $k$-nearest-neighbor predictor with -0.088 . Clearly, the true conditional expectation for the stochastic Lorenz system has nonadditive structure that the additive autoregressive predictor is not able to capture. Unlike the stochastic logistic map, we see that the $k$-nearest-neighbor predictor gave out-of-sample mean-squared errors better than the lower bound computed using $\hat{h}$. This indicates that the estimates of $\hat{\bar{h}}(4)$ have a non-negligible positive bias even when $T=10^{4}$ and that the predictive mean is easier to estimate than a functional of the entire predictive density.

\section{CONCLUSIONS}

In this paper, we have developed an information-theoretic model selection procedure for determining the optimal model order for prediction of a subcomponent of a stochastic dynamical system. The procedure provides a fully nonparametric and automatic estimator of the negative log-predictive likelihood, which can be minimized to determine the optimal model order. We demonstrated that minimizing the entropy rate is, conceptually, exactly the complement of maximizing the active information storage. Despite this conceptual complementarity, the entropy rate-based procedure resulted in a consistent estimator of the optimal model order, while the 
active information storage-based procedure could be either consistent or inconsistent depending on the system under consideration. Finally, we demonstrated how the model selection routine can be paired with an information-theoretic bound on the mean-squared error of a predictor of the dynamical system to determine the quality of a set of candidate predictors.

Our results advise additional caution when using model selection routines based on fixed tuning parameters. For example, a fixed, typically small $k$ is often used to select the embedding dimension through the mean-squared error of a $k$-nearest-neighbor predictor, a method sometimes called the Ragwitz criterion after Ref. [26] and implemented in two of the main toolboxes for information-theoretic estimation [35,63]. As noted by others, the value of $k$ (or, equivalently, the size of the bandwidth for a kernel regression) can be used as a proxy for how "deterministic" a dynamical system is Refs. [64,65], and a small value of $k$ should only be used for a system that can be considered operationally deterministic, that is, that can be modeled as a stochastic dynamical system with very small (or zero) dynamical noise. Additional experiments, not reported here, indicate that like the fixed- $k$ active information storage criterion, the fixed- $k$ mean-squared error criterion performs poorly for stochastic dynamical systems with non-negligible dynamical noise. The Ragwitz criterion is especially popular in information dynamics studies in neuroscience [66-69]. Given the mixed evidence for strong determinism in electrophysiological measurements [64,70-73], caution should be taken in applying the fixed- $k$ mean-squared error criterion in that domain.

Model selection via the negative log-predictive likelihood should prove valuable to the general problem of attractor reconstruction via a delay-coordinate embedding. As argued in Ref. [74], a great deal of attractor reconstruction studies occur in reverse: Fine-grained details such as attractor dimensions, correlation integrals, and Lyapunov exponents are sometimes used to determine the appropriate embedding dimension for a delay-coordinate embedding. The former, a model inference problem, is decidedly more difficult than the latter, a model selection problem. We have already seen how, for a stochastic dynamical system, the combination of these two problems leads to suboptimal results with the active information storage model selection criterion. Thus, the negative log-predictive likelihood may be used for model selection before performing the additional model inference tasks. For example, we took this approach in Sec. IV in estimating the entropy rate for a stochastic dynamical system. A similar approach could be applied in selecting the model order for nonlinear state filters $[75,76]$ and estimators of dynamical coupling $[77,78]$.

We have not addressed the problem of selecting the time delay for optimal prediction of a continuous-time stochastic dynamical system. A natural way to incorporate a time delay into the problem of optimal prediction is to consider a predictive horizon, that is, to consider the predictive density of a $q$-distant future $X_{t+q}$ given the most recent past $\left\{X_{s}, s<\right.$ $t$ \} for some $q \geqslant 0$. With $q$ fixed at the desired predictive horizon for the system, the time delay $\tau$ may be determined by minimizing the negative log-predictive likelihood of $X_{t+q}$ given $\left\{X_{t-j \tau}\right\}_{j=1}^{\ell}$ simultaneously over $\ell$ and $\tau>0$. We leave the investigation of this approach for future work.
We have demonstrated the utility of the negative logpredictive likelihood for order estimation of stochastic dynamical systems with relatively small Markov order. More work needs to be done to determine how this procedure behaves for non-Markovian stochastic dynamical systems: Does it select a "good-enough" model order relative to some criterion of "good enough," e.g., a model order that balances between bias introduced from too small a model order and variance introduced from too large a model order? Moreover, an estimate of the sample size requirement necessary to consistently estimate a given model order would be desirable, similar to the results in Refs. [74,79] demonstrating the sample size requirement for using a leave-one-out cross-validated mean-squared error criterion to determine the model order is approximately quadratic in the model order.

\section{ACKNOWLEDGMENTS}

I thank Paul Rapp and Christopher Cellucci for valuable discussions related to this work. I acknowledge support from the Uniformed Services University and the Defense Medical Research and Development Program.

\section{APPENDIX: ENTROPY RATE AND ACTIVE INFORMATION STORAGE FOR THE SETAR MODEL}

The specific entropy rate for the $\operatorname{SETAR}(2 ; 2,2)$ process (21) is given by

$$
\begin{aligned}
h\left[X_{t} \mid X_{t-1}=\right. & \left.x_{t-1}, X_{t-2}=x_{t-2}\right] \\
= & I_{(-\infty, 3.25]}\left(x_{t-2}\right) h\left[\epsilon_{-, t}\right] \\
& +I_{(3.25, \infty)}\left(x_{t-2}\right) h\left[\epsilon_{+, t}\right]
\end{aligned}
$$

and thus the entropy rate is given by

$$
\begin{aligned}
h\left[X_{t} \mid X_{t-1}, X_{t-2}\right]= & P\left(X_{t-2} \leqslant 3.25\right) h\left[\epsilon_{-, t}\right] \\
& +P\left(X_{t-2}>3.25\right) h\left[\epsilon_{+, t}\right] .
\end{aligned}
$$

Rather than approximate the density of $X_{t-2}$ as we did with the stochastic logistic map, we approximate $P\left(X_{t-2} \leqslant 3.25\right)$ by

$$
\hat{P}\left(X_{t-2} \leqslant 3.25\right)=\frac{1}{T} \sum_{t=1}^{T} I\left[X_{t} \leqslant 3.25\right]
$$

using a length $T=10^{6}$ realization, giving $\hat{P}\left(X_{t-2} \leqslant 3.25\right)=$ 0.706 . Substituting into (A2) gives $\bar{h}(\infty)=\bar{h}(2) \approx-0.14$. Finally, to approximate the active information storage, we estimate $h\left[X_{t}\right]$ using the $k$ th-nearest-neighbor estimator (25) with $k=5$ with a length $T=10^{6}$ realization, giving $\hat{h}\left[X_{t}\right]=$ 0.87 and thus $\mathcal{A}(\infty)=\mathcal{A}(2) \approx \hat{h}\left[X_{t}\right]-\hat{\bar{h}}(2)=1.01$. 
[1] N. H. Packard, J. P. Crutchfield, J. D. Farmer, and R. S. Shaw, Phys. Rev. Lett. 45, 712 (1980).

[2] F. Takens, Lect. Notes Math. 898, 366 (1981).

[3] H. Kantz and T. Schreiber, Nonlinear Time Series Analysis, Vol. 7 (Cambridge University Press, Cambridge, 2004).

[4] E. Bradley and H. Kantz, Chaos 25, 097610 (2015).

[5] G. Sugihara, B. Grenfell, R. M. May, P. Chesson, H. Platt, and M. Williamson, Phil. Trans. R. Soc. Lond. B 330, 235 (1990).

[6] A. Garfinkel, M. L. Spano, W. L. Ditto, and J. N. Weiss, Science 257, 1230 (1992).

[7] Y. Jian, S. Silvestri, J. Brown, R. Hickman, and M. Marani, Ecol. Appl. 26, 2611 (2016).

[8] C. Frazier and K. Kockelman, Transp. Res. Rec. 1897, 9 (2004).

[9] P. Shang, X. Li, and S. Kamae, Chaos Solitons Fractals 25, 121 (2005).

[10] M. De Domenico, A. Lima, and M. Musolesi, Pervasive Mob. Comput. 9, 798 (2013).

[11] V. Sreekumar, S. Dennis, I. Doxas, Y. Zhuang, and M. Belkin, PLoS ONE 9, e97166 (2014).

[12] J. Garland and E. Bradley, in International Symposium on Intelligent Data Analysis (Springer, Berlin, 2013), pp. 210-222.

[13] T. Sauer, J. A. Yorke, and M. Casdagli, J. Stat. Phys. 65, 579 (1991).

[14] A. Eftekhari, H. L. Yap, M. B. Wakin, and C. J. Rozell, Phys. Rev. E 97, 022222 (2018).

[15] J. D. Farmer and J. J. Sidorowich, Phys. Rev. Lett. 59, 845 (1987).

[16] J. P. Crutchfield and B. S. McNamara, Complex Syst. 1, 121 (1987).

[17] M. Casdagli, Physica D 35, 335 (1989).

[18] G. Sugihara and R. M. May, Nature 344, 734 (1990).

[19] A. S. Weigend and N. A. Gershenfeld, in Time Series Prediction: Forecasting the Future and Understanding the Past, Santa Fe Institute Studies in the Science of Complexity, Vol. XV, edited by N. A. Gershenfeld and A. S. Weigend (Addison-Welsey, Reading, MA, 1993).

[20] J. Garland, R. G. James, and E. Bradley, Phys. Rev. E 93, 022221 (2016).

[21] M. Casdagli, S. Eubank, J. D. Farmer, and J. Gibson, Physica D 51, 52 (1991).

[22] S. Ørstavik and J. Stark, Phys. Lett. A 247, 145 (1998).

[23] J. Stark, Nonlinear Dynamics and Statistics (Birkhäuser, Boston, MA, 2001), pp. 81-103.

[24] K.-S. Chan and H. Tong, Chaos: A Statistical Perspective (Springer Science \& Business Media, New York, 2013).

[25] B. Cheng and H. Tong, J. R. Statist. Soc. B 54, 427 (1992).

[26] M. Ragwitz and H. Kantz, Phys. Rev. E 65, 056201 (2002).

[27] G. Claeskens and N. Hjort, Model Selection and Model Averaging (Cambridge University Press, Cambridge, 2008), pp. 22-65 and 70-241.

[28] J. T. Lizier, M. Prokopenko, and A. Y. Zomaya, Inf. Sci. 208, 39 (2012).

[29] H. Tong, Non-Linear Time Series: A Dynamical System Approach (Oxford University Press, Oxford, 1990), pp. 152 and 178.

[30] S. Caires and J. A. Ferreira, Stat. Inf. Stoch. Process. 8, 151 (2005).

[31] K.-S. Chan and H. Tong, Bernoulli 8, 1 (2002).

[32] J. P. Crutchfield and D. P. Feldman, Chaos 13, 25 (2003).

[33] R. W. Yeung, IEEE Trans. Inf. Theor. 37, 466 (1991).
[34] A. Kraskov, H. Stögbauer, and P. Grassberger, Phys. Rev. E 69, 066138 (2004)

[35] J. T. Lizier, Front. Robot. AI 1, 11 (2014).

[36] W. Gao, S. Oh, and P. Viswanath, in 2017 IEEE International Symposium on Information Theory (ISIT) (IEEE, Los Alamitos, CA, 2017), pp. 1267-1271.

[37] E. N. Lorenz, J. Atmos. Sci. 26, 636 (1969).

[38] D. Darmon, Entropy 18, 190 (2016).

[39] Z. Lincheng and L. Zhijun, Acta Math. Sin. 1, 314 (1985).

[40] J. Fan and Q. Yao, Nonlinear Time Series: Nonparametric and Parametric Methods (Springer Science \& Business Media, New York, 2003).

[41] J. A. Nelder and R. Mead, Comput. J. 7, 308 (1965).

[42] M. Box, Comput. J. 8, 42 (1965).

[43] S. G. Johnson, The nlopt nonlinear-optimization package, version 2.4.2, http://ab-initio.mit.edu/nlopt.

[44] L. Devroye, L. Gyorfi, A. Krzyzak, and G. Lugosi, Ann. Stat. 22, 1371 (1994).

[45] D. Darmon, sidpy: Python Tools for Specific Information Dynamics, https://doi.org/10.5281/zenodo.1186296.

[46] B. F. Finkenstädt, Q. Yao, and H. Tong, Stat. Comp. 11, 229 (2001).

[47] D. Holstein and H. Kantz, Phys. Rev. E 79, 056202 (2009).

[48] L. Wasserman, All of Statistics: A Concise Course in Statistical Inference (Springer Science \& Business Media, New York, 2013).

[49] K.-S. Chan and H. Tong, J. R. Statist. Soc. B 56, 301 (1994).

[50] M. Korzen and S. Jaroszewicz, J. Stat. Soft. 57, 1 (2014).

[51] H. Tong and K.-S. Lim, J. R. Statist. Soc. B 42, 245 (1980).

[52] P. A. P. Moran, Aust. J. Zool. 1, 291 (1953).

[53] T. M. Cover and J. A. Thomas, Elements of Information Theory (John Wiley \& Sons, New York, 2012), p. 663.

[54] H. Singh, N. Misra, V. Hnizdo, A. Fedorowicz, and E. Demchuk, Am. J. Math. Manage. Sci. 23, 301 (2003).

[55] R. Chen and R. S. Tsay, J. Am. Stat. Assoc. 88, 955 (1993).

[56] S. N. Wood, J. Am. Stat. Assoc. 99, 673 (2004).

[57] R. McGill, J. W. Tukey, and W. A. Larsen, Am. Stat. 32, 12 (1978).

[58] J. D. Hart, J. R. Statist. Soc. B 53, 173 (1991).

[59] E. N. Lorenz, J. Atmos. Sci. 20, 130 (1963).

[60] P. Grassberger and I. Procaccia, Physica D 9, 189 (1983).

[61] J. Stark, D. S. Broomhead, M. E. Davies, and J. Huke, J. Nonlinear Sci. 13, 519 (2003).

[62] A. Rößler, SIAM J. Numer. Anal. 48, 922 (2010).

[63] M. Lindner, R. Vicente, V. Priesemann, and M. Wibral, BMC Neurosci. 12, 119 (2011).

[64] M. Casdagli, J. R. Statist. Soc. B 54, 303 (1992).

[65] Q. Yao and H. Tong, Physica D 115, 49 (1998).

[66] F. Roux, M. Wibral, W. Singer, J. Aru, and P. J. Uhlhaas, J. Neurosci. 33, 17827 (2013).

[67] P. Wollstadt, M. Martínez-Zarzuela, R. Vicente, F. J. Díaz-Pernas, and M. Wibral, PLoS ONE 9, e102833 (2014).

[68] C. Gómez, J. T. Lizier, M. Schaum, P. Wollstadt, C. Grützner, P. Uhlhaas, C. M. Freitag, S. Schlitt, S. Bölte, R. Hornero et al., Front. Neuroinform. 8 (2014)

[69] G. Gómez-Herrero, W. Wu, K. Rutanen, M. C. Soriano, G. Pipa, and R. Vicente, Entropy 17, 1958 (2015). 
[70] C. A. Skarda and W. J. Freeman, Behav. Brain Sci. 10, 161 (1987).

[71] W. S. Pritchard, D. W. Duke, and K. K. Krieble, Psychophysiology 32, 486 (1995).

[72] J. Theiler and P. E. Rapp, Electroencephalogr. Clin. Neurophysiol. 98, 213 (1996).

[73] R. G. Andrzejak, K. Lehnertz, F. Mormann, C. Rieke, P. David, and C. E. Elger, Phys. Rev. E 64, 061907 (2001).

[74] B. Cheng and H. Tong, Phil. Trans. R. Soc. Lond. A 348, 325 (1994).
[75] F. Hamilton, T. Berry, and T. Sauer, Phys. Rev. X 6, 011021 (2016)

[76] F. Hamilton, T. Berry, and T. Sauer, The European Physical Journal Special Topics 226, 3239 (2017).

[77] T. Schreiber, Phys. Rev. Lett. 85, 461 (2000).

[78] D. Darmon and P. E. Rapp, Phys. Rev. E 96, 022121 (2017).

[79] B. Cheng and H. Tong, in Stochastic and Spatial Structures of Dynamical Systems: Proceedings of the Colloquium, edited by S. van Strien and S. Verduyn Lunel (Koninklijke Nederlandse Akademie van Wetenschappen, Amsterdam, 1996), pp. 29-37. 